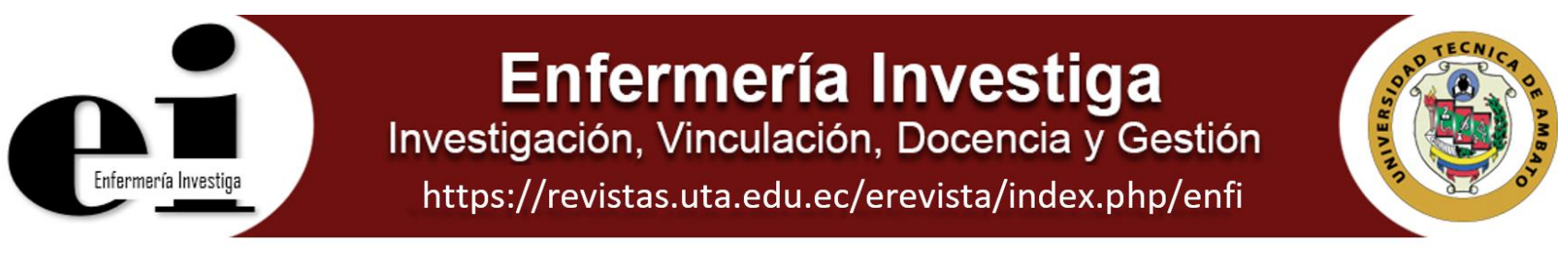

\title{
LIDERAZGO ENFERMERO
}

\section{NURSING LEADERSHIP}

\author{
Mercedes Gómez del Pulgar García-Madrid, PhD1, orcid:http://orcid.org/0000-0002-0418-2337
}

${ }^{1}$ Directora del Centro de Educación Superior Hygiea adscrito a la Universidad a Distancia de Madrid (UDIMA). Madrid-España. Departamento de Enfermería Fisioterapia y Podología. Universidad Complutense. Madrid-España.

Autor de correspondencia: Mercedes Gómez del Pulgar García-Madrid, PhD, correo electrónico: mgp@hygiea.es

2477-9172 / 2550-6692 Derechos Reservados @ 2021 Universidad Técnica de Ambato, Carrera de Enfermería. Este es un artículo de acceso abierto distribuido bajo los términos de la Licencia Creative Commons, que permite uso ilimitado, distribución y reproducción en cualquier medio, siempre que la obra original es debidamente citada.

Recibido: 05 de octubre 2021

Aceptado: 20 de noviembre 2021

El liderazgo ha estado presente en las civilizaciones de todas las épocas, y tanto las organizaciones como la sociedad en su conjunto son conscientes de la importancia de su papel (1), este concepto ya no es descrito únicamente como una característica individual, sino como una dinámica global y compleja (2). En definitiva, es un fenómeno poliédrico, producto de la interacción entre diversos factores (personales, interpersonales y contextuales) (1).

Durante el siglo pasado, la investigación sobre liderazgo se focalizó en su función para desarrollar y poner en práctica la innovación (3). Actualmente se observa un interés creciente por el liderazgo y su desarrollo entre los profesionales sanitarios, con especial énfasis en formas de liderazgo de equipo, participativo, el coaching y el liderazgo auténtico (1). Se han publicado infinidad de aproximaciones, enfoques y definiciones de liderazgo "hay tantas definiciones del concepto de líder como personas han intentado definirlo" (4). De todos los enfoques y definiciones que se pueden encontrar en la literatura es interesante hacer referencia al enfoque que realiza Bass (5) sobre liderazgo transformacional, diferenciándolo del liderazgo transaccional.

El liderazgo transaccional es ese estilo de liderazgo en el que existe un intercambio entre el líder y sus seguidores, donde estos reciben un valor a cambio de su trabajo, es decir existe una relación consto-beneficio. El liderazgo transformacional, en contraposición, es aquel que motiva a las personas a hacer más de lo que ellas mismas esperan y como consecuencia, se producen cambios en los grupos, en las organizaciones y en la sociedad, que representan a su vez un beneficio colectivo, es decir, el liderazgo supone un compromiso con el logro de la misión de una organización, dejando de lado los intereses personales y orientarse a cumplir los objetivos colectivos. Esta visión del liderazgo tiene relación con las necesidades humanas, y específicamente con las que se ubican en el dominio del crecimiento personal, autoestima y autorrealización.

En el ámbito de la salud en general y al de la Enfermería en particular, es uno de los enfoques que se aproxima a lo que debería motivar un líder enfermero, dado que los líderes transformacionales, a través de la influencia ejercida en sus interrelaciones con los miembros del grupo, estimulan cambios de visión que conducen a cada individuo a dejar de lado sus intereses particulares para buscar el interés colectivo, aun cuando no tenga satisfechas sus necesidades vitales existenciales. Pero, ¿A qué se refiere cuando se habla de liderar? Liderar no debe confundirse con ordenar, mandar, ni con dirigir desde el poder que te otorga una posición, que te permite llevar a cabo un cargo. Liderar tiene que ver con inspirar, motivar y movilizar cambios en las personas. El liderazgo enfermero puede realizarse desde cualquier ámbito en el que estos profesionales desempeñan sus funciones: en los cuidados con los pacientes, en la promoción de la salud y en la prevención de la enfermedad, pero también debe aplicarse en las actividades de gestión, administración, investigación, innovación, programas docentes emprendimiento y política (6).

Si lo que se busca es conseguir un objetivo, es necesario que el líder sea capaz de conseguir que quien debe colaborar para la consecución de ese objetivo, se implique y esté dispuesto a seguir lo que propone. Para esto es importante poseer una serie de características que harán que el liderazgo suceda. La profesional de enfermería llamado a liderar debe ser competente, conocer muy bien su ámbito de actuación. No sería coherente que pretendiera liderar una nueva forma de entender la enfermería alguien que no conociera la disciplina y que no tuviera una competencia reconocida en este campo. Esa competencia debe estar sustentada en un adecuado conocimiento y una probada experiencia.

Además, un líder enfermero debe tener una visión inspiradora e ilusionante que motive y movilice para que, incluso en situaciones difíciles, sea capaz de hacer que le sigan con confianza y con la esperanza de que dicha situación se va a resolver. Si no hay una visión compartida, no hay liderazgo. El Profesional de enfermería, como líder, debe poseer además carácter para tomar decisiones, seguridad, honestidad, integridad, coherencia, debe ser esa persona que busca hacer siempre lo que dice, que da ejemplo con su comportamiento y conecta con los demás de manera genuina, desde la humildad, la empatía y el humanismo. De esta manera la implicación y el compromiso de las personas se gana, no se impone. Todo esto puede resumirse en ser buena persona, en ir más allá de satisfacer las propias necesidades, el propio ego, la ambición o la avaricia; para enfocarse en liderar 
desde el servicio a los demás. Porque a corto plazo, no serlo puede tener resultados, pero a largo plazo el talento se escapa, huye de jefes ingratos, de personas que no tratan bien a los demás $(7,8)$.

Algunos ejemplos de líderes enfermeros los encontramos en las referencias históricas; así, y a modo de ejemplo, recordamos a Florence Nightingale como "La dama de la lámpara"; Mary Seacole como "La enfermera que nadie recuerda" (9), Irena Sendler (10), también llamada "El ángel del Gueto de Varsovia", quien durante la Segunda Guerra Mundial ayudó y salvó a más de dos mil quinientos niños judíos prácticamente condenados a ser víctimas del Holocausto, arriesgando su propia vida, Virginia Henderson y sus "14 necesidades básicas", Isabel Zendal (11), la primera enfermera que se embarcó en misión internacional en la Real Expedición Filantrópica de la Vacuna con el Dr. Balmis.

Pero, además, encontramos lideres enfermeros que reúnen las características, mencionadas previamente, en nuestro día a día. Por ejemplo, quien no se ha encontrado con a esa enfermera, mentora de prácticas, que no tiene "cargo", esa enfermera que es líder para sus pacientes, que confían en ella, que habla con la autoridad que da la experiencia y que es una fuente de aprendizaje para los estudiantes y compañeros; o esa supervisora admirada por su cercanía, entrega y humildad, que es capaz de transmitir tranquilidad y seguridad en las situaciones más críticas y que sabe conseguir lo mejor de cada una de las personas del equipo, valorando sus cualidades, capacidades y apreciando las opiniones discordantes desde el respeto y puesta en valor de las mismas en beneficio del equipo y de las metas.

Los profesionales de enfermería representan la mayor profesión sanitaria y por lo tanto, pueden y deben jugar un papel integral en la planificación del futuro de la atención de salud. El Consejo Internacional de Enfermeras (CIE) estableció el lema del día internacional de la Enfermera en el año 2021 como "Enfermería: una voz para liderar. Una visión de futuro para la atención de salud" (12).

Los profesionales de Enfermería están llamados a liderar, desde todos y cada una de las esferas en las que desarrolla su labor, ámbito asistencial, docente, investigador y de gestión. Desde el ámbito asistencial, se debe ejercer el liderazgo en los cuidados al paciente, en la promoción de la salud y la prevención de la enfermedad; desde el ámbito docente, debemos ser líderes para los estudiantes y futuros enfermeros; desde la investigación, debemos buscar y compartir las mejores evidencias, motivar a participar y a impulsar investigaciones que aporten resultados innovadores para mejorar la salud de la población, y por supuesto liderar desde los puestos de gestión, administración, emprendimiento y política (6).

Un buen liderazgo se logra a través de programas de formación innovadores en esta temática y proyectos de investigación que permitan favorecer la calidad de los servicios, la satisfacción de los usuarios y la reducción de costes asociados (13), para acelerar el fortalecimiento del liderazgo de las enfermeras se proponen algunas recomendaciones, entre las que se encuentran cambiar la percepción de la Enfermería como 'ciencia blanda' y elevar el estatus y perfil de enfermería en el sector sanitario; abordar la segregación laboral por género y eliminar la percepción de enfermería como 'trabajo de mujeres; eliminar la discriminación laboral en función del género o la maternidad, crear autoconfianza y obtener preparación para asumir posiciones de liderazgo, asegurar ambientes de trabajo seguros y sensibles a la conciliación de trabajo y familia, dar oportunidades de acceso a financiación para el desarrollo del liderazgo, formación superior u otros roles profesionales, fomentar mayor acceso a las redes profesionales y a mentoría. Todo esto implica fomentar el crecimiento y la participación personal, desarrollar habilidades en todos los miembros de la organización, impulsar la creatividad (14). Así mismo, desde las organizaciones enfermeras, los líderes deben señalar el camino del devenir de la profesión, simbolizar y representar el sentido y los valores de la organización, promover y facilitar que los profesionales compartan sus ideales e involucrarles en la gestión, en definitiva, hacer que éstos se sientan orgullosos de su profesión y de su organización.

En definitiva, y según Ferrer C. (15), tener en cuenta a las enfermeras supone un triple impacto: se mejora la salud de los ciudadanos, se mejora la igualdad, porque somos mayoritariamente mujeres y se aportan mejoras económicas a la sociedad, porque los sistemas son más eficientes. Existe una necesidad real de visibilizar los cuidados porque en palabras de esta autora, "Las enfermeras trabajamos en la trastienda del sistema" La biotecnología, el diagnóstico de las enfermedades, el tratamiento...todo eso es muy visible, pero no así los cuidados. Sin embargo, cuando una persona requiere cuidados complejos, es importante contar con un profesional, y ese profesional es la enfermera".

\section{REFERENCIAS}

1. Sánchez J.A., López E., Del Llano J.E. Liderazgo de organizaciones y equipos sanitarios.Cuadernos de Gestión Sanitaria №5. Fundación Gaspar Casal-Almirall. 2018. Disponible en: https://www.actasanitaria.com/documentos/lainnovacion-en-la-gestion-de-las-organizaciones-sanitarias-cuaderno-de-f-gaspar-casal-y-almirall/

2. Serrano Orellana BJ, Portalanza Ch A, Serrano Orellana BJ, Portalanza Ch A. Influenciadel liderazgo sobre el clima organizacional. Suma Negocios [Internet]. 2014;5(SPE11):117-125. Disponible http://www.scielo.org.co/scielo.php?script=sci_arttext\&pid=S2215910X2014000200117\&lng=en\&nrm=iso\&tlng=es

3. Danet A, García Romera I, March Cerdá JC. Liderazgo transformacional en las organizaciones sanitarias. Una revisión bibliográfica. Rev Comun y Salud RCyS. 2016;6(1):81-95. Disponible en: https://dialnet.unirioja.es/servlet/articulo?codigo=5786978\&info=resumen\&idioma=SPA

4. Guevara E. Evolución del liderazgo. De los gurús a los farsantes. E-Book HWCG Editores.2020. Disponible en: https://www.amazon.com/-/es/Emeterio-Guevaraebook/dp/B08DN3RP5W?asin=B08DN3RP5W\&revisionld=\&format=2\&depth=1

5. Leadership and Performance Beyond Expectations", by Bernard M. Bass (Book Review) ProQuest. Disponible en: https://www.proquest.com/openview/76c51c9c9dd6c9be026f434caea3479e/1?pqorigsite $=$ gscholar $\&$ cbl $=1818075$

6. Zabalegui A. A propósito del liderazgo en enfermería. Nursing (Lond) [Internet]. 2018;35(1):6. Disponible en: 
https://www.elsevier.es/es-revista- nursing-20-articulo-a-proposito-del-liderazgo-enfermeria-S0212538218300013

7. Balderas A. Reinventa tu liderazgo. ESIC editorial. Madrid 2021 Disponible en:https://www.meet-in.es/paraliderar-a-largo-plazo-hay-que-ser-buenapersona/?fbclid=IwAR2MZrgXaNQXEvOly6xMPajCzsKfyEG5Tj8CKLG8H80fDIIO-y- 0V6eWGR8

8 Howard Gardner, "Una mala persona no llega nunca a ser buen profesional”Disponible en: https://www.lavanguardia.com/lacontra/20160411/401021583313/una-mala-persona- no-llega-nunca-a-serbuen-profesional.html

9. Mary Seacole (1805-1881): La enfermera que nadie recuerda | Ciencia y más | Mujerescon ciencia. Disponible en: https://mujeresconciencia.com/2019/05/23/mary-seacole-1805-1881-la-enfermera-que- nadie-recuerda/

10. Irena Sendler, el "Ángel de Varsovia" National Geographic. [Internet]. Disponible en: https://historia.nationalgeographic.com.es/a/irena-sendler-angel- varsovia 16761

11. Isabel Zendal Gómez | Real Academia de la Historia [Internet]. Disponible en: https://dbe.rah.es/biografias/52862/isabel-zendal-gomez

12. El Consejo Internacional de Enfermeras anuncia el tema del Día Internacional de la Enfermera 2021. Disponible en: www.icnvoicetolead.com.

13. Pinzón O. Liderazgo en los servicios de salud. Cienc y Salud Virtual [Internet]. 2014;6(1):37-53. Disponible en:https://revistas.curn.edu.co/index.php/cienciaysalud/article/view/413

14. Perdomo Y, Prieto R. El liderazgo como herramienta de competitividad para la gerencia del servicio. CICAG Rev del Cent Investig Ciencias Adm y Gerenciales. 2009;6(2):30-48. Disponible en: https://dialnet.unirioja.es/servlet/articulo?codigo=3153331\&info=resumen\&idioma=SPA

15. Ferrer C. Liderazgo Enfermero: Pinceladas de experiencia. Ed. Tecnos. Madrid. 2021. Disponible en: https://www.tecnos.es/ficha.php?id=6646435\&pageid=4

16. Perdomo Y, Prieto R. El liderazgo como herramienta de competitividad para la gerencia del servicio. CICAG Rev del Cent Investig Ciencias Adm y Gerenciales, ISSN-e 1856-6189. 2009;6(2):30-48. Disponible en: https://dialnet.unirioja.es/servlet/articulo?codigo=3153331\&info=resumen\&idioma=SPA

17. Ferrer C. Liderazgo Enfermero: Pinceladas de experiencia. Ed. Tecnos. Madrid. 2021. Disponible en: https://www.tecnos.es/ficha. php?id=6646435\&pageid=4 